\title{
Creencias sobre el uso del condón en una población universitaria
}

\author{
Abril Valdez, Elba; Román Pérez, Rosario; Cubillas Rodríguez, María José y \\ Domínguez Ibáñez, Sandra Elvia \\ Creencias sobre el uso del condón en una población universitaria \\ CIENCIA ergo-sum, vol. 25, núm. 3, noviembre 2018-febrero 2019|e27 \\ Universidad Autónoma del Estado de México, México
}

Esta obra está bajo una Licencia Creative Commons Atribución-NoComercial-SinDerivar 4.0 Internacional.

Abril Valdez, E.; Román Pérez, R.; Cubillas Rodríguez, M. J. y Domínguez Ibáñez, S. E. (2018). Creencias sobre el uso del condón en una población universitaria. CIENCIA ergo-sum, 25(3). https://doi.org/10.30878/ ces.v25n3a5 


\section{Creencias sobre el uso del condón en una población universitaria}

\section{Beliefs on condom use in students university}

\section{Elba Abril Valdez}

Centro de Investigación en Alimentos y Desarrollo, México

abril@ciad.mx

Recepción: 26 de abril de 2017

Aprobación: 15 de agosto de 2017

Rosario Román Pérez

Centro de Investigación en Alimentos y Desarrollo, México

rroman@ciad.mx

Maria José Cubillas Rodriguez

Centro de Investigación en Alimentos y Desarrollo, México

mjcubillas@ciad.mx

Sandra Elvia Dominguez Ibáñez

Centro de Investigación en Alimentos y Desarrollo, México

dominguez@ciad.mx

\section{Resumen:}

Se describen y analizan creencias acerca del uso del condón en jóvenes universitarios con o sin antecedentes de relaciones sexuales. Se aplicó una encuesta a 1513 estudiantes, hombres y mujeres seleccionados al azar mediante muestreo probabilístico. Del total, 62\% reportó haber tenido relaciones sexuales y $38 \%$ dijo que no. Las creencias sobre el uso del condón fueron asociadas con la pérdida del placer, las dificultades de uso y los estereotipos de género, más por los varones que por las mujeres. Se concluye que en estudiantes universitarios persisten creencias poco favorecedoras acerca del uso del condón aun cuando no hayan iniciado relaciones coitales. De no promover cambios, la tendencia se mantendrá con sus consecuentes riesgos.

Palabras Clave: uso de condón, sexualidad, jóvenes universitarios.

\section{Abstract:}

The study describes and analyzes beliefs about condom use among university students with/ without a history of sexual intercourse. A survey was applied to 1513 students, men and women randomly selected by probabilistic sampling. Sixty two percent reported having ever had sex and $38 \%$ said no. Beliefs about condom use were associated with loss of pleasure, difficulties of use, and gender stereotypes, more by men than by women. It is concluded that in college students persist beliefs not conducive to condom use even if they have not initiated coital relationships and do not promote changes, the trend will remain with its consequent risks.

KEYWORDS: condom use, sexuality, university students.

\section{INTRODUCCIÓN}

El condón o preservativo masculino es un método anticonceptivo de barrera que bien utilizado evita embarazos no deseados y es, junto con el condón femenino, un método efectivo para prevenir el contagio de VIH e infecciones de transmisión sexual (ITS). Pese a la difusión sobre los métodos de barrera, la información que tienen los y las jóvenes suele ser sesgada o insuficiente, por lo que ejercen su sexualidad sin la debida protección y, al incorporarla como parte de su sistema de creencias, se arraiga dando lugar a comportamientos de riesgo (Osorio y Cuello, 2013). Esto genera un desconocimiento que se reproduce en creencias sobre la vida sexual, las cuales son transmitidas de una generación a otra.

Las creencias forman parte de la estructura del pensamiento de una persona que se configuran en función de lo aceptable o no dentro de su contexto social (Azjen y Fishben, 1980). Son preceptos heredados y muy arraigados que se manifiestan como información y opiniones más allá de si corresponden a una condición 
real o imaginaria. Entre esas creencias se pueden mencionar las relacionadas con la pérdida del placer y el hecho de que una mujer que solicita a su pareja usar condón sea señal de un comportamiento promiscuo, entre otras (Pérez et al., 2011). Este tipo de opiniones conlleva a una vida sexual menos cuidadosa y por ende propensa a riesgos como la transmisión de enfermedades sexuales y embarazos no planificados, que traen como consecuencia un impacto psicosocial a mediano y largo plazo traducido en el aumento de los índices de deserción escolar, aumento del número de hijos, relaciones disfuncionales y reducción de ingresos (Osorio y Cuello, 2013).

Entre los hombres mexicanos el uso del condón tiene poca aceptación; asimismo, es muy poco utilizado por las mujeres debido a las diferencias sociales y culturales que existen entre ambos. El tema tiene que ver con la construcción social de las masculinidades sustentadas sobre la condición de riesgo (De Keijzer, 1997). Estudios realizados con jóvenes señalan que los hombres tienen un patrón de comportamiento sexual más riesgoso que el de las mujeres, ya que en ellos es mayor el porcentaje que inicia su vida sexual a temprana edad y tiene un número más grande de parejas (Álvarez et al., 2006; Moral de la Rubia, 2007; Restrepo et al., 2015). Sin embargo, es mayor el porcentaje de mujeres que reportan no usar condón con sus parejas en sus relaciones sexuales, lo que indica que ellas, en cierta medida, pueden estar en riesgo alto de contraer VIH o cualquier otra ITS (García et al., 2005; Tschann et al., 2010; De JesúsReyes y Menkes, 2014; Uribe et al., 2015; INEGI, 2016). Entre los factores que posibilitan esta inconsistencia en el uso de condón se encuentra el poder en la toma de decisiones en el ámbito sexual donde el hombre es quien decide qué se va hacer y cómo se va hacer (Enríquez et al., 2005; Robles et al., 2006; Camacho y Pabón, 2014).

En el caso de Sonora hay poca información al respecto, por lo que es pertinente documentar el tema dada su relevancia en relación con la salud sexual de sectores vulnerables como el de la población joven. Lo anterior es parte de construcciones culturales sobre la sexualidad. En México predominan aún los valores y principios de una sociedad patriarcal, donde se organizan las relaciones sexuales con base en atributos y estereotipos asignados a cada sexo. La doble moral y la permisividad sexual otorgada socialmente a los hombres sugiere la marginación de las mujeres en el acto sexual (Amuchástegui, 1998; Montesinos, 2002). La poca educación sobre la sexualidad que existe en México propicia y ha perpetuado graves y serios problemas tanto en el campo de la salud personal como en el social. Tratándose de jóvenes, estas ideas son aún más tradicionales porque están basadas en un ideal de lo que es ser mujer y hombre, desligado del ejercicio de su sexualidad, y como si fueran ajenos al placer (Saeteros et al., 2015; Hernández y González, 2016).

En la sociedad sonorense existe una forma hegemónica de socializar que les brinda ventajas y coloca a las mujeres en desventaja en relación con las decisiones que toman, lo cual repercute en su salud sexual. Este tipo de comportamientos tiende a estereotiparse y, hasta cierto punto, es visto como normal, a unque tienen un costo para la salud de hombres y mujeres (Gutiérrez, 2006; Salguero, et al., 2016). Entre los factores que mantienen vigentes las prácticas sexuales desprotegidas, los mitos ocupan un lugar relevante, así como la información negativa no sustentada científicamente sobre los métodos de protección. En el caso del condón, es común pensar que se trata tan sólo de un mecanismo de control natal que, al mismo tiempo, tiene la desventaja de disminuir el placer de la relación sexual en el varón, además de que se le considera un instrumento de planificación familiar que no suele tener mucha efectividad. El hecho de que al condón se le considere un método de control natal puede influir en la decisión de usarlo. En este sentido, debe tenerse en cuenta que el ejercicio sexual entre jóvenes sin vida marital tiene que ver más con el erotismo y el deseo, que con planear la formación de una familia. En la mayoría de las ocasiones los encuentros sexuales se tienen sin haberse planeado, y en lo que menos se piensa es en protegerse, como lo reportan jóvenes embarazadas de un estudio realizado en el norte de México (Román, 2000). Por otro lado, los mensajes que reciben de diferentes medios hacen parecer a la actividad sexual como algo casual, de forma espontánea y romántica, libre de riesgo y responsabilidades (Vargas et al., 2006; Pérez et al., 2011).

En un estudio realizado con estudiantes universitarios en México, con una muestra aleatoria de 355 mujeres entre 18 y 26 años, se evaluaron conocimientos, autoeficacia y actitudes relacionadas con el VIH. 
Además, se incluyeron tres variables situacionales que hacen referencia a la historia sexual de la pareja al tener sexo vaginal, oral y anal. Se reportó que la única variable predictora del uso inconsistente del condón fue la creencia de que éste disminuye la satisfacción sexual (Robles et al., 2006). Resultados similares reporta Moral de la Rubia (2007) en un estudio descriptivo que se realizó en una universidad del noreste de México. Se trabajó con una muestra de 395 universitarios en la que 54\% de los hombres y $30 \%$ de las mujeres reportó haber tenido sexo. El uso de preservativo fue predicho sólo por su uso en la primera relación sexual y con menor frecuencia en relaciones posteriores. Entre los factores que redujeron el uso de condón estuvo la valoración de las consecuencias sexuales negativas sobre su empleo (pérdida de espontaneidad durante el acto) y creencias sin una base objetiva. Los resultados de los dos estudios concluyen que el no uso del condón está asociado con la reducción del placer durante la relación sexual. En otros casos se piensa que entorpece el desarrollo espontáneo del coito porque su uso exige controlar, pensar, detenerse, esperar. Desde este punto de vista, el preservativo se ve como un objeto extraño que se inmiscuye y se vive como una imposición, incluso como represión del deseo sexual. De acuerdo con Olavarría (2001), los conocimientos sobre la sexualidad se estructuran en una serie de mitos y de ocultamientos que enfatizan lo biológico y reproductivo y aplazan todo aquello que tenga que ver con el placer. Así, al mismo tiempo que los jóvenes ejercen y viven su sexualidad, específicamente las relaciones coitales, dichas prácticas son consideradas como irresponsables por la sociedad. Una prueba de ello es que se les juzga con severidad cuando, por alguna razón, las mujeres terminan embarazadas o cuando ellos o ellas adquieren algún tipo de infección de transmisión sexual, como el VIH.

Si bien existen diversos trabajos que analizan el uso del condón en jóvenes de universidad, por su misma complejidad la información que hay es aún insuficiente para documentar las diferentes creencias que la juventud tiene a este respecto, ya que se centran sobre todo en jóvenes que tienen vida sexual activa sin tomar en cuenta que dichas creencias pueden estar presentes también en aquellos jóvenes que aún no tienen relaciones sexuales con su pareja (Uribe et al., 2012; Osorio y Cuello, 2013; De Jesús-Reyes y Menkes, 2014; Camacho y Pabón, 2014). De ahí que el objetivo de este artículo sea analizar las creencias sobre el uso del condón que prevalecen en las y los jóvenes universitarios, independientemente de si han tenido relaciones coitales o no, considerando que éstas pueden condicionar de alguna manera su decisión de usarlo durante su primera relación sexual.

\section{Materiales y método}

\section{1. Participantes}

Participaron 1513 estudiantes seleccionados a través de muestreo estratificado (Cochran, 1990) con un nivel de confianza de $95 \%$ y un margen de error de 0.5 , de una población total de 21316 jóvenes de una universidad pública en Hermosillo, Sonora, México. El porcentaje de mujeres fue de $51.9 \%$ (786) y el de hombres 48.1\%(727), el cual está distribuido en 32 licenciaturas agrupadas en seis divisiones académicas de acuerdo con lo r eportado por la institución (cuadro 1). La media de edad para ambos sexos fue de 20 años con un rango de 17 años mínimo y 29 años como máximo. 
CUADRO 1

Distribución de la muestra

\begin{tabular}{|lccc}
\hline \multirow{2}{*}{ Licenciaturas por división académica } & \multicolumn{3}{c}{ Sexo } \\
\cline { 2 - 4 } & Hombre & Mujer & Total \\
\hline Humanidades y Bellas Artes & 65 & 103 & 168 \\
Ciencias Económicas y Administrativas & 122 & 168 & 290 \\
Ciencias Exactas y Naturales & 71 & 31 & 102 \\
Ciencias Sociales & 107 & 298 & 405 \\
Ciencias Biológicas y de la Salud & 85 & 121 & 206 \\
Ingenierías & 277 & 65 & 342 \\
\hline Total & 727 & 786 & 1513 \\
\hline
\end{tabular}

Fuente: Dirección de Planeación. Elaborado con base en datos proporcionados por la Dirección de Servicios Escolares, Universidad de Sonora.

\subsection{Instrumento}

Para recolectar la información se aplicó un cuestionario que incluía preguntas sobre aspectos sociodemográficos, historia de vida sexual (edad de inicio vida sexual, número de parejas, uso de condón, entre otros), además de una escala para evaluar las creencias sobre el uso del condón. Dicha escala se retomó de la Encuesta Instrumental de Salud Reproductiva diseñada por la Organización Panamericana de la Salud (OPS, 2000), la cual está conformada por 78 reactivos que exploraban diferentes aspectos relacionados con la salud reproductiva. Sin embargo, para fines de este estudio sólo se retomaron las 20 afirmaciones que evalúan las creencias sobre el uso del condón. Los enunciados fueron evaluados a partir de cuatro opciones de respuesta, donde total acuerdo es el de menor valor hasta total desacuerdo con mayor valor.

\subsection{Procedimiento}

El instrumento utilizado fue autoaplicado durante horario de clase, previa autorización del personal docente y el consentimiento informado de estudiantes. La participación fue voluntaria y confidencial. El análisis de la información se realizó con el paquete estadístico Statistical Package for the Social Sciences (SPSS), versión 18. Se obtuvieron medidas descriptivas y para el analizar las diferencias entre grupos se utilizó $t$ de student.

A partir del análisis factorial exploratorio se buscó reconocer la estructura que pudiera tener en los y las jóvenes las creencias sobre el uso del condón. El resultado del análisis muestra que la consistencia interna del instrumento en general es aceptable al obtener 0.777 en el modelo de Alfa de Cronbach (Oviedo y Campo Arias, 2005). A partir del análisis factorial los reactivos de la escala se agruparon en cuatro componentes y por tipo de variables que los componían. Quedaron de la siguiente manera:

a) Creencias relacionadas con la pérdida del placer, tales como "el condón reduce el placer sexual “, "a las mujeres usar condón les corta el deseo", "a la mayoría de las mujeres no les gusta usar condón”, “a la mayoría de los hombres no les gusta usar condón”, “a los hombres usar condón les corta el deseo" y "el hombre disfruta más si no usa condón”.

b) Las creencias relacionadas con sentimientos que dificultan su uso se agruparon en ítems que tienen que ver con complicaciones: "los condones son complicados de usar", "los condones requieren que la pareja tenga autocontrol", "es difícil discutir el uso del condón con la posible pareja", "da vergüenza comprar condones" y "da vergüenza pedir condones en las clínicas”. 
c) Creencias sobre las ventajas de usarlo: "usar condón con una nueva pareja es buena idea", "las mujeres deberían de pedir a sus parejas que usen condón" y "los condones permiten disfrutar más por la seguridad que dan".

d) En las creencias estereotipadas de género se agruparon aquellas que tienen que ver con estereotipos de género: "las mujeres que piden a su pareja usar condón tienen muchas parejas", "usar condón no es de hombres", "no es necesario usar condón si uno conoce a su pareja", "durante la relación sexual es responsabilidad del hombre usar condón", "sólo los homosexuales deben usar condón” " “los hombres que piden a su pareja usar condón tienen muchas parejas”.

\section{Resultados}

\section{1. Comportamiento sexual}

De los participantes, $62 \%$ reportó haber tenido relaciones sexuales alguna vez en su vida y $38 \%$ dijo que no. En el cuadro 2 se observa que más hombres (69.3\%) que mujeres (55\%) reportaron haber tenido alguna vez relaciones coitales.

\section{CUADRO 2}

Porcentaje de participantes con y sin actividad sexual

\begin{tabular}{|lcccc}
\hline \multirow{2}{*}{ Actividad sexual } & \multicolumn{2}{c}{ Hombres } & \multicolumn{2}{c}{ Mujeres } \\
\cline { 2 - 5 } & Frecuencia & Porcentaje & Frecuencia & Porcentaje \\
\hline Sí & 504 & 69.3 & 432 & 55 \\
No & 219 & 30.1 & 354 & 45 \\
Sin respuesta & 4 & 0.6 & - & - \\
\hline Total & 727 & 100 & 786 & 100 \\
\hline
\end{tabular}

Fuente: elaboración propia.

En relación con quienes dijeron haber tenido relaciones sexuales, hombres y mujeres muestran diferencias estadísticamente significativas en edad de inicio de vida sexual, número de parejas sexuales y en frecuencia de uso de condón (cuadro 3). La edad promedio de inicio de vida sexual fue de 17.10 años en la primera relación sexual (rango 10-25 años) para los varones y 18.18 años (con rango de 14-29 años) para las mujeres (cuadro 3).

\section{CUADRO 3}

Diferencias entre hombres y mujeres respecto a sus prácticas sexuales

\begin{tabular}{|llccc}
\hline Variable & Sexo & $N^{* *}$ & Media & Significancia \\
\hline Edad debut sexual & Hombre & 495 & 17.08 & $0.000^{*}$ \\
Númer & 422 & 481 & 18.18 & 2.03 \\
$\begin{array}{l}\text { sexuales en el } \\
\text { último año }\end{array}$ & Hombre & 398 & 1.17 & $0.000^{*}$ \\
$\begin{array}{l}\text { Frecuencia en uso } \\
\text { de condón durante }\end{array}$ & Hombre & 473 & 2.15 & $0.002^{*}$ \\
relación sexual & Mujer & 391 & 2.43 & \\
\hline
\end{tabular}

Fuente: elaboración propia.

Nota: ${ }^{*} p \leq 0.05 .{ }^{* *}$ El número de $N$ varía por las no respuestas obtenidas en el cuestionario.

El número de parejas sexuales durante el último año fue mayor en los varones, pero fueron los menos quienes dijeron usar siempre condón durante sus relaciones sexuales. Las diferencias fueron estadísticamente 
significativas (cuadro 3). El rango para los varones fue de 1 a 17 parejas y las mujeres de 1 a 8 parejas sexuales durante el último año.

En relación con el uso del condón, en este grupo más hombres (90\%) que mujeres (78.3\%) dijeron haber usado condón durante su primera relación sexual. De éstos, $67.5 \%$ de los hombres y $61.1 \%$ de las mujeres dijo que lo usó durante su última relación sexual (cuadro 4).

\section{CUADRO 4}

Uso de condón durante primera y última relación sexual

\begin{tabular}{|lrrrrrrrc}
\hline & \multicolumn{3}{c}{ Primera relación sexual } & \multicolumn{3}{c}{ Ultima relación sexual } \\
\cline { 2 - 10 } Uso de condón & \multicolumn{2}{c}{ Hombres } & \multicolumn{2}{c}{ Mujeres } & \multicolumn{2}{c}{ Hombres } & \multicolumn{2}{c}{ Mujeres } \\
\cline { 2 - 10 } & \multicolumn{1}{c}{ Fr** } & $\%$ & Fr** & $\%$ & Fr** & $\%$ & Fr** & $\%$ \\
\hline Sí & 453 & 90 & 335 & 78.3 & 334 & 67.5 & 256 & 61.1 \\
No & 40 & 8 & 81 & 18.9 & 155 & 31.3 & 159 & 37.9 \\
No recuerdo & 9 & 2 & 12 & 2.8 & 6 & 1.2 & 4 & 1 \\
\hline Total & 502 & 100 & 428 & 100 & 495 & 100 & 419 & 100 \\
\hline
\end{tabular}

Fuente: elaboración propia.

Nota: ${ }^{* *}$ El número de $N$ varía por las no respuestas obtenidas en el cuestionario.

En relación con quién llevó el condón durante la primera relación sexual, hombres y mujeres coincidieron en reportar que "los dos lo habían llevado" (hombres $84.4 \%$ y mujeres $73.9 \%$ ). Sin embargo, este porcentaje varió al preguntarles sobre su última relación sexual: 79.6\% de los varones dijo que ellos lo habían llevado y las mujeres dijeron que su pareja (69.3\%). La mujer sigue manteniéndose al margen en cuanto a tomar la iniciativa de ser ella la que lleve el condón. Tanto en la primera como en la última relación el porcentaje se mantuvo bajo (6.1\% y 8.15\%). No obstante, no deja de ser relevante el hecho de que algunas jóvenes reporten haber tomado la iniciativa.

El condón masculino junto con el femenino son los únicos métodos de barrera que pueden prevenir el VIH y las ITS. El no uso implica un mayor riesgo de adquirir algún padecimiento; sin embargo, el condón no es visto por los y las jóvenes como un método de prevenir el riesgo de estas enfermedades, pues lo consideran más como un método anticonceptivo. Durante la primera relación sexual sólo $6.4 \%$ de los varones y $0.6 \%$ de las mujeres lo usó teniendo en mente que es una medida preventiva de ITS o VIH. Situación similar se observó durante su última relación sexual (cuadro 5).

\section{CUADRO 5}

Razones para usar condón

\begin{tabular}{|lrrrrrrrr}
\hline \multirow{2}{*}{ ¿Qué querías prevenir? } & \multicolumn{3}{c}{ Primera relación sexual } & \multicolumn{3}{c}{ Ultima relación sexual } \\
\cline { 2 - 9 } & \multicolumn{2}{c}{ Hombres } & \multicolumn{1}{c}{ Mujeres } & \multicolumn{1}{c}{ Hombres } & \multicolumn{2}{c}{ Mujeres } \\
\cline { 2 - 9 } & Fr** & \multicolumn{1}{c}{$\%$} & \multicolumn{1}{c}{ Fr* } & \multicolumn{1}{c}{$\%$} & Fr** & $\%$ & Fr** & $\%$ \\
\hline Embarazo & 109 & 30.2 & 76 & 22.8 & 132 & 35.9 & 94 & 32.2 \\
ITS & 23 & 6.4 & 2 & 0.6 & 26 & 7.1 & 4 & 1.4 \\
Las dos & 226 & 62.6 & 265 & 76.6 & 206 & 56 & 192 & 65.8 \\
Nada & 3 & 0.8 & 0 & 0 & 4 & 1.1 & 2 & 0.7 \\
\hline Total & 361 & 100 & 334 & 100 & 368 & 100 & 292 & 100 \\
\hline
\end{tabular}

Fuente: elaboración propia.

Nota: ${ }^{* *}$ El número de $N$ varía por las no respuestas obtenidas en el cuestionario.

Respecto a los motivos expresados por los y las jóvenes para no usar condón se engloban principalmente en la incertidumbre y la no planificación de las relaciones sexuales. Más hombres (14.9\%) que mujeres (10.4\%) dijeron que "tener relaciones sexuales no fue planificado"; por tanto, no iban preparados con condón. Le sigue "no haber pensado usar condón” (13.9\% de los varones vs. 8.6\% de las mujeres). De igual modo, pensar 
que no se corre ningún riesgo fue ligeramente mayor en los varones (4.4\%) respecto a las mujeres (3.2\%). Cabe señalar que en menores porcentajes, pero también importante, las mujeres mencionaron la negativa de la pareja a usarlo, la vergüenza de ellas para comprarlos, el temor de los varones de no sentir lo mismo, entre otros, lo cual podría confirmar la persistencia de estereotipos y creencias que no favorecen la práctica de usar condón durante las relaciones sexuales.

\section{LAS CREENCIAS SOBRE EL USO DEL CONDÓN}

Se utilizó el análisis ANOVA para probar la relación entre el ejercicio sexual y los cuatro componentes en los que se agrupó la escala de creencias. El nivel de significancia se estableció en $p<0.05$. Los hallazgos del análisis mostraron que estadísticamente hay diferencias en tres de las categorías. Los varones tienen una tendencia a estar, en su mayoría, de acuerdo con la pérdida de placer, las dificultades de su uso y las creencias estereotipadas de género del uso del condón en comparación con las mujeres (cuadro 6). Fueron más los varones que mujeres los que dijeron estar de acuerdo en aquellas afirmaciones que tienen que ver con la pérdida de placer con el uso del condón.

\section{CUADRO 6}

\begin{tabular}{|c|c|c|c|c|}
\hline Variable & Sexo & $N$ & Media & Significancia \\
\hline \multirow{2}{*}{$\begin{array}{l}\text { Creencias relacionadas con la pérdida } \\
\text { de placer }\end{array}$} & Hombre & 489 & 2.55 & \multirow{2}{*}{$0.00 *$} \\
\hline & Mujer & 411 & 2.79 & \\
\hline \multirow{2}{*}{ Creencias estereotipadas de género } & Hombre & 496 & 3.43 & \multirow{2}{*}{$0.00 *$} \\
\hline & Mujer & 417 & 3.56 & \\
\hline \multirow{2}{*}{$\begin{array}{l}\text { Creencias relacionadas con } \\
\text { sentimientos que dificultan su uso }\end{array}$} & Hombre & 499 & 3.26 & \multirow{2}{*}{$0.06^{*}$} \\
\hline & Mujer & 420 & 3.11 & \\
\hline \multirow{2}{*}{$\begin{array}{l}\text { Creencias relacionadas con las ventajas } \\
\text { de usarlo }\end{array}$} & Hombre & 196 & 1.77 & \multirow{2}{*}{$0.05^{*}$} \\
\hline & Mujer & 416 & 1.67 & \\
\hline
\end{tabular}

Creencias sobre el uso del condón en hombres y mujeres con actividad sexual Fuente: elaboración propia.

Nota: ${ }^{*} p \leq 0.05$

En cuanto a creencias estereotipadas de género fueron los varones quienes mostraron valores superiores en la media en comparación con las mujeres al estar en mayor acuerdo con afirmaciones como que "las mujeres que piden a su pareja usar condón tiene muchas parejas" o "no es necesario usar condón si uno conoce a su pareja". En relación con las creencias sobre las ventajas de usarlo, fueron las mujeres quienes dijeron estar en mayor acuerdo con afirmaciones como "usar condón con una persona nueva es buena idea", "las mujeres deberían pedir a sus parejas que usen condón” y "los condones permiten disfrutar más por la seguridad que dan”. Respecto a las creencias relacionadas con sentimientos que dificultan el uso del condón (cuadro 6) las diferencias observadas no fueron significativas; sin embargo, cabe señalar que fueron las mujeres quienes dijeron estar en mayor acuerdo con afirmaciones tales como "usar condón con una nueva pareja es buena idea", "las mujeres deberían de pedir a sus parejas que usen condón", entre otras. Por otro lado, también se exploraron diferentes creencias sobre el uso de condón que pudieran presentarse entre las y los jóvenes que reportaron no tener vida sexual activa respecto a los que dijeron que sí la tenían. Los datos muestran diferencias estadísticamente significativas en dos componentes: en las creencias relacionadas con la pérdida de placer y creencias relacionadas con sentimientos que dificultan su uso (cuadro 7). 
CUADRO 7

Creencias sobre el uso del condón en participantes con y sin actividad sexual

\begin{tabular}{|c|c|c|c|c|}
\hline Variable & Actividad sexual & $\bar{N}$ & Media & Significancia \\
\hline \multirow{2}{*}{$\begin{array}{l}\text { Creencias relacionadas con la } \\
\text { pérdida de placer }\end{array}$} & Sí & 901 & 2.66 & \multirow{2}{*}{$0.000^{*}$} \\
\hline & No & 444 & 2.90 & \\
\hline \multirow{2}{*}{$\begin{array}{l}\text { Creencias estereotipadas de } \\
\text { género }\end{array}$} & Sí & 914 & 3.49 & \multirow{2}{*}{$0.172 *$} \\
\hline & No & 478 & 3.45 & \\
\hline \multirow{2}{*}{$\begin{array}{l}\text { Creencias relacionadas con } \\
\text { sentimientos que dificultan su uso }\end{array}$} & Sí & 920 & 3.19 & \multirow{2}{*}{$0.000^{*}$} \\
\hline & No & 484 & 2.82 & \\
\hline \multirow{2}{*}{$\begin{array}{l}\text { Creencias relacionadas con las } \\
\text { ventajas de usarlo }\end{array}$} & Sí & 913 & 1.73 & \multirow{2}{*}{$0.313^{*}$} \\
\hline & No & 462 & 1.70 & \\
\hline
\end{tabular}

Fuente: elaboración propia.

Nota: ${ }^{*} p \leq 0.05$

Los y las jóvenes que reportaron haber tenido relaciones sexuales tuvieron una tendencia a estar más de acuerdo con aquellas afirmaciones que tienen que ver con la pérdida del placer por el uso del condón que quienes dijeron no haber tenido relaciones sexuales. Lo anterior podría explicarse por la experiencia, pero es importante remarcar la existencia de este tipo de creencias aun cuando no se ha tenido actividad sexual. Por otro lado, se observaron diferencias significativas estadísticamente en creencias relacionadas con sentimientos que dificultan su uso. La media fue superior entre los y las jóvenes que dijeron haber tenido relaciones sexuales, lo que indica que estuvieron más en desacuerdo con las afirmaciones que tienen que ver con las dificultades para el uso del condón. El caso contrario es en los jóvenes que reportaron no tener relaciones sexuales, pues son quienes muestran un mayor acuerdo en este tipo de afirmaciones aun sin haber vivido la experiencia de usarlos.

\section{Discusión}

Los resultados muestran que los varones inician su vida sexual a más temprana edad que las mujeres. El resultado concuerda con lo reportado por Moreno et al. (2008), Instituto Mexicano de la Juventud (2012) y Gutiérrez et al. (2012). Este comportamiento tiene que ver con la conformación de la masculinidad y con el ejercicio de la sexualidad a temprana edad en los varones como variable que les permite conformar socialmente lo que es ser hombre (Connell, 1987; Olivarría, 2001). Lo anterior deviene en la permisividad social sobre esta actividad en los varones y en poca aceptación en las mujeres pese a la presencia de discursos liberadores y de igualdad (Gutiérrez, 2006; Motesinos, 2002). Los jóvenes fortalecen su masculinidad a partir del ejercicio de su sexualidad, tal como la sociedad hegemónica les señala y les refuerza. Los comportamientos tales como inicio de vida sexual a edad temprana, mayor número de parejas, entre otros, van conformando en el joven su masculinidad (Olavarría, 2001; Aguirre y Güell, 2002). Así, socialmente al varón se le felicita, se le facilita y se le alienta a que ejerza su sexualidad; en cambio, a la mujer se le reprime y castiga.

En cuánto al uso del condón en este grupo, el análisis mostró una baja notable en la última relación sexual en comparación con el uso del condón durante la primera relación sexual. Este descenso en la utilización del preservativo en la primera relación sexual está relacionado probablemente con los significados culturales que en nuestra sociedad tiene el preservativo, tales como que disminuye el placer, que le corta espontaneidad al acto, entre otros (Robles et al., 2006; Moral de la Rubia, 2007). Estudios realizados en México señalan que cerca de $40 \%$ de los y las jóvenes no utilizan ningún método anticonceptivo durante su primera relación sexual. Esta proporción aumenta cuando la relación no fue planeada, lo cual puede acarrear consecuencias como embarazos no deseados, VIH e ITS (Moreno et al., 2008; Rivera et al., 2013).

En relación con quién lleva el condón durante la relación sexual, los datos muestran que la mujer se mantiene al margen en cuanto a tomar la iniciativa en el uso del condón, lo cual pudiera atribuirse a la 
connotación social que se le suele adjudicar a las mujeres, ya que normalmente no es bien visto ni es lo que se espera que hagan y podría poner en entre dicho su pureza. El varón por tradición es el que lleva el control de la relación sexual -es su rol asignado-. Si una mujer toma la iniciativa y sugiere usar el condón a su pareja, podría ponerle en entredicho su masculinidad y el control sobre la relación (Amuchástegui, 1998). Además, socialmente se espera que la mujer sea fiel y llegue virgen al matrimonio, por lo que el hecho de llevarlo la haría sospechosa de ya haber tenido relaciones sexuales. La situación se asume diferente para los hombres, quienes deben llegar con mucha experiencia al matrimonio si no quieren ser humillados $\mathrm{u}$ opacados por su mujer, e incluso por otros hombres (Sevilla, 2008).

Por otro lado, los resultados dan cuenta de que hombres y mujeres comparten una preocupación significativa por el embarazo producto de una relación sexual. El uso del condón entre los y las participantes gira alrededor de evitar embarazos, más que prevenir una posible enfermedad. Según la sociedad, el embarazo en este periodo es incompatible con la idea de jóvenes que estudian una carrera universitaria, conscientes de la existencia de un plan de vida en el que representaría un retardo para conseguir la meta. A esto se suma el peso de las reglas culturales que conciben la idea de una juventud no sexuada, en la que no es válido tener relaciones sexuales y mucho menos ser padres. El embarazo es la muestra fehaciente de que se es sexualmente activa en una edad que no es aceptado. Se espera que durante esta etapa de la vida hombres y mujeres se preparen para ingresar a la vida adulta y posteriormente formar una familia (Guevara, 2010).

A pesar de la aparente apertura a los cambios en pro de la igualdad de género en el terreno sexual, siguen prevaleciendo en las y los participantes los estereotipos que favorecen de cierta forma a los varones, de ahí que la decisión de usar o no condón durante la relación sexual no se toma pensando en prevenir el riesgo de VIH o ITS, sino más bien en prevenir un posible embarazo. Este panorama nos habla de que la sexualidad y su vigilancia sigue cargada hacia la reproducción. Es evidente que persiste un esquema de doble moral que otorga mayor control sobre la propia sexualidad a los varones y se resta autonomía y poder de decisión a las mujeres. Así, un mismo comportamiento tiene significado diferente dependiendo del sexo biológico, tal es el caso del número de parejas sexuales. Para los varones un mayor número de parejas sexuales les da seguridad, los empodera de su masculinidad y les da estatus entre sus pares y la sociedad en general. En cambio, a las mujeres esa misma condición las lleva a perder control con su pareja en el ejercicio de su sexualidad, disminuye la probabilidad de negociar el uso del condón ante la posibilidad de que se ponga en duda su fidelidad y se pierda la confianza.

En relación con las creencias sobre el uso del condón, el análisis mostró que fueron más varones que mujeres los que manifestaron estar más de acuerdo con aquellas afirmaciones relacionadas con la pérdida de placer al usar el condón. Estas diferencias podrían estar vinculadas con la connotación cultural diferenciada de la sexualidad en hombres y mujeres. Ambos reciben mensajes y valores diferentes para el ejercicio de su sexualidad por parte de la sociedad. Es distinto lo que a cada uno le es permitido o prohibido hacer o sentir durante las relaciones coitales. Así, a los varones les es permitido sentir y disfrutar de su cuerpo y el de las mujeres con libertad, mientras que en ellas la sexualidad sigue ligada a la reproducción y la consideran ajena a su placer (Montesinos, 2002, Rocha y Díaz Loving, 2005; Rocha, 2008). Las y los jóvenes construyen alrededor de estas creencias y estereotipos estilos, modos o maneras de relacionarse con su pareja, así como la manera en que manifiestan y expresan su sexualidad y cómo cuidan de ella.

Este tipo de creencias relacionadas con el uso el condón repercute en las prácticas sexuales de los jóvenes. Los pares, la familia, la universidad, la religión y los medios de comunicación son elementos de su contexto inmediato que refuerzan este tipo de creencias y no favorecen el cuidado de la salud. Los resultados muestran que estas creencias también están presentes entre las y los jóvenes que declaran no tener vida sexual activa. Estas ideas preconcebidas podría ser un factor que influyera en ellos y ellas a la hora de decidir si usan o no condón en su primera relación sexual. Independientemente de que se tenga o no experiencia coital, la idea de "no placer" con condón está presente en las y los universitarios. 


\section{CONCLUSIONES}

Los datos hasta aquí reportados confirman la permanencia de creencias relacionadas con la permisividad sexual que existen para el varón. En los hombres traer un condón y usarlo durante la relación sexual es aceptado como normal o "natural". En las mujeres este comportamiento resulta inapropiado porque cuestiona su fidelidad, lo que no sucede con los varones, ya que se interpreta como un acto responsable.

Por otro lado, en el imaginario de los jóvenes aún prevalecen creencias que les lleva a no utilizar condón durante sus relaciones sexuales, y en el de las mujeres a mantenerse temerosas por exigirlo a su pareja. Se hace visible lo persistente de las creencias de una población enmarcada en un modelo patriarcal que logra permear incluso en aquellos jóvenes que aún no viven la experiencia de la relación sexual, a pesar de ser universitarios, más informados y con posibilidades de tomar decisiones de manera más objetiva, lo que puede influir en su vida sexual futura y en exposición a riesgos en su salud.

\section{ANÁLISIS PROSPECTIVO}

Los datos que se obtuvieron en este estudio permiten identificar la necesidad de crear intervenciones dirigidas a las y los jóvenes con el fin de sensibilizarlos para desmitificar creencias, actitudes, mitos y tabúes relacionados con el uso del condón y otros comportamientos sexuales que repercuten en su salud sexual y les impiden tomar decisiones de manera objetiva. Las acciones deben de ir más allá de sólo proporcionar información sobre sexualidad y métodos anticonceptivos. Es pertinente brindar educación sexual con perspectiva de género a fin de que las jóvenes principalmente tengan una participación más activa en la toma de decisiones de una manera responsable e informada.

Las estrategias de acción son a nivel discurso en donde se enfatiza la necesidad de políticas públicas que promuevan prácticas saludables en el área de la sexualidad; sin embargo, las acciones realizadas se enfatizan en la prevención de embarazos. Es decir, la sexualidad sigue ligada a la reproducción y ajena a los varones. No se aborda con una visión de género que permita promover la salud sexual con responsabilidad compartida en ambos sexos.

En este sentido, quedan interrogantes por resolver y que permitirían tener un panorama más completo de la problemática. ¿Qué pasa con los y las jóvenes que no están incluidos en la población objetivo? ¿Sus creencias en cuanto al uso del condón también están permeadas por estereotipos de género? Si bien la pregunta queda abierta para futuras investigaciones, podemos asumir a partir del contexto regional que la tendencia sería similar a lo expresado por los y las jóvenes universitarias según lo corroboran datos de INEGI (2016). Los resultados señalan que 62.3\% de las mujeres jóvenes de 15 a 29 años han iniciado su vida sexual; de ellas, una de cada dos no utilizó un método anticonceptivo durante su primera relación sexual, además de que siete de cada diez nacimientos ocurridos en el trienio 2011-2013 se dieron entre las mujeres de 15 a 29 años de edad. Lo anterior podría poner en evidencia que los programas de prevención no han impactado a la población objetivo, por lo que las estrategias deberían replantearse a fin de incidir de manera positiva en las y los jóvenes. De seguir abordándose como hasta la fecha se ha hecho, los embarazos no planeados y las infecciones por vía sexual seguirán la misma tendencia o se elevarán.

\section{REFERENCIAS}

Aguirre, R. y Güell, P. (2002). Hacerse hombres. La construcción de la masculinidad en los adolescentes y sus riesgos. Washington, D. C.: Organización Panamericana de la Salud. Organización Mundial de la Salud. UNFPA. Fundación W. K. Kellogg. ASDI. Disponible en http://www1.paho.org/Spanish/HPP/HPF/ADOL/Hacerse \%20hombres1.pdf 
Álvarez, L., Calero, R. y León E. (2006). Salud sexual y reproductiva desde el punto de vista del varón. Revista Cubana de Salud Pública, 32(1). Disponible en http://scielo.sld.cu/scielo.php? script $=$ sci_arttext\&pid=S0864-34662006000100004\&lng=es.

Amuchástegui, A. (1998). La dimensión moral de la sexualidad y de la virginidad en las culturas híbridas mexicanas. Relaciones, 74(19),103-13.

Ajzen, I. y Fishbein, M. (1980). Understanding attitudes and predicting social behavior. Englewood Cliffs: PrenticeHall.

Camacho, D. y Pabón, Y. (2014). Percepciones que afectan negativamente el uso del condón en universitarios de la Costa Caribe colombiana. Hacia promoción de la salud, 19(1), 54-67.

Cochran, W. (1990). Técnicas de muestreo. México: CECSA.

Connell, R. W. (1987). Gender and power: Society, the person and sexual politics. Cambridge: Polity Press.

De Jesús-Reyes, D. y Menkes, C. (2014). Prácticas y significados del uso del condón en varones adolescentes de dos contextos de México. Papeles de población, 73-97.

De Keijzer, B. (1997). El varón como factor de riesgo. Masculinidad, salud mental y salud reproductiva, en Esperanza Tuñón (coord.), Género y salud en el sureste de México (pp. 67-81). México: UJAT/ECOSUR

Enríquez, D., Sánchez, R. y Robles, S. (2005). Variables relativas al uso del condón en hombres y mujeres universitarios. Revista Electrónica de Psicología Iztacala, 8(2), 81-98.

García, E., Fernández, P. y Rico, R. (2005). Género y sexo como variables moduladoras del comportamiento sexual en jóvenes universitarios. Psicothema, 17(1), 49-56.

Guevara, E. (2010). Cuando el amor se instala en la modernidad: intimidad, masculinidad y jóvenes en México. México: Universidad Autónoma de México.

Gutiérrez, S. (2006). Género y masculinidades: relaciones y prácticas culturales. Revista Ciencias Sociales, 111-112, 155-175.

Gutiérrez, P., Rivera-Dommarco, J., Shamah-Levy, T., Villalpando-Hernández, S., Franco, A., Cuevas-Nasu, L., Romero-Martínez, M. y Hernández-Avila, M. (2012). Encuesta Nacional de Salud y Nutrición 2012. Resultados Nacionales. México: Instituto Nacional de Salud Pública.

Hernández, A. y González, J. (2016). Los roles y estereotipos de género en los comportamientos sexuales de jóvenes de Coahuila, México: aproximación desde la Teoría Fundamentada. CIENCIA ergo-sum, 23. Disponible en http:// www.redalyc.org/articulo.oa?id=10446094003. ISSN 1405-0269.

INEGI (Instituto Nacional de Estadística y Geografía). (2016). Estadísticas a propósito del día internacional de la juventud (15 a 29 años) 12 de agosto. Boletín día de la juventud 2016. México.

Instituto Mexicano de la Juventud (2012). Encuesta Nacional de Valores en Juventud 2012. Centro de Investigación y Estudios sobre Juventud en México. México. Disponible en http://www.imjuventud.gob.mx/imgs/uploads/ ENVAJ_2012.pdf

Montesinos, R. (2002). Sexualidad y erotismo: la sexualidad en la perspectiva de la práctica de hombres y mujeres. Las rutas de la masculinidad: ensayos sobre el cambio cultural y el mundo moderno. Barcelona: Gedisa.

Moral de la Rubia, J. (2007). Conducta sexual y uso de preservativo en estudiantes universitarios. Medicina Universitaria, 9(37), 173-180.

Moreno, D., Rivera, P., Robles, S., Barroso, R., Frías, B. y Rodríguez, M. (2008). Características del debut sexual de los adolescentes y determinantes del uso consistente del condón desde el análisis contingencial. Psicología y Salud, $18(2), 207-225$.

Olavarría, J. (2001). ¿Hombres a la deriva? Poder, trabajo y sexo. Chile: Flacso.

Organización Panamericana de la Salud (OPS). (2000). Protocolos de investigación para el estudio de la salud sexual y reproductiva de los adolescentes y hombres jóvenes en América Latina. División de Promoción y Protección de la Salud. Programa de Familia y Población. Washington, D.C. Disponible en http://cidbimena.desastres.hn/ docum/ops/pdf/protocol.pdf 
Osorio, L. y Cuello, K. (2013). Evaluación de mitos sexuales de acuerdo al género y nivel de formación en jóvenes universitarios y de educación media. Realitas, Revista de Ciencias Sociales, Humanas y Artes, 1, 9-13.

Oviedo, H. C. y Campo-Arias, A. (2005). Aproximación al uso del coeficiente alfa de Cronbach. Revista Colombiana de Psiquiatría, 34(4), 572-580. Disponible en http://www.redalyc.org/articulo.oa?id=80634409

Pérez, D., Fonseca, C. y Sánchez, J. (2011). Placer y decisión: reflexiones sobre las causas por las cuales los jóvenes universitarios no utilizan el condón a pesar de estar informados. Revista Digital Universitaria, 12(6). Disponible en http://www.revista.unam.mx/vol.12/num6/art62/index.html

Restrepo, K., Toro, A. y Cardona, J. (2015). Prácticas sexuales y uso del preservativo en adolescentes escolarizados de Medellín, 2014. Biomedicina, 1(2). DOI: 10.3823/5001

Rivera, A., Caballero, P., Pérez I. y Montero, L. (2013). Distrés psicológico, género y conductas de riesgo. Universitas Psichologica, 12(1), 105-118. Disponible en http://revistas.javeriana.edu.co/index.php/revPsycho/ article/view/879

Robles, S., Piña, J. y Moreno, D. (2006). Determinantes del uso inconsistente del condón en mujeres que tienen sexo vaginal, oral o anal. Anales de Psicología, 22(2), 200-2004.

Rocha, T. (2008). Cultura de género y sexismo: de Díaz-Guerrero al posmodernismo. Entnopsicología Mexicana: siguiendo la huella teórica y empírica de Díaz Guerrero. México: Trillas.

Rocha, T. y Díaz Loving, R. (2005). Cultura de género: la brecha ideológica entre hombres y mujeres. Anales de Psicología, 21(1), 42-49.

Román, R. (2000).Del primer vals alprimer bebé. Vivencias del embarazo en las jóvenes. México: Secretaría de Educación Pública, Instituto Mexicano de la Juventud.

Saeteros, R., Díaz, Z., Sanabria Ramos, G. y Pérez, J. (2015). Representaciones socioculturales sobre el ideal de la salud sexual y reproductiva en estudiantes universitarios ecuatorianos. Revista Cubana de Salud Pública, 41(3), 459-475.

Salguero M., Soriano M. y Ayala C. (2016) Estereotipos de género: sexualidad y anticoncepción en jóvenes universitarios de clase media. Investigaciones Feministas, 7(1), 335-352.

Sevilla, T., (2008). Sexo inseguro. Un análisis de la racionalidad como parte del riesgo entre jóvenes caleños y caleñas. Revista Latinoamericana de Ciencias Sociales, Niñez y Juventud, 1(6), 257-294.

Tschann J., Flores, E., de Groat, C., Deardorff, J. y Wibbelsman C. (2010). Condom negotiation strategies and actual condom use among latino youth. Journal of Adolescent Health, 47, 254-262.

Uribe, J., Aguilar, J., Zacarías, X. y Aguilar, A. (2015). Modelos explicativos del uso del condón en las relaciones sexuales de adolescentes. Acta de Investigación Psicológica-Psychological Research Records, 1904-1915.

Uribe, J., Amador, G., Zacarías, X. y Villarreal, L. (2012). Percepciones sobre el uso del condón y la sexualidad entre jóvenes. Revista Latinoamericana de Ciencias Sociales, Niñez y Juventud, 10(1), 481-494.

Vargas, E., Barrera, F., Burgos, M. y Daza, B. (2006). La intención de los jóvenes de tener relaciones sexuales en la adolescencia: el papel de la televisión, la relación con los padres y las cogniciones. Universitas Psychologica, 5(1), 69-84.

\section{BY-NC-ND}

